\title{
THE NEED FOR AN EVIDENCE-BASE IN MOBILE TECHNOLOGY IN MEDICINE
}

\author{
Dr Rahul Chakrabarti MBBS ${ }^{1,2}$ \\ ${ }^{1}$ Editor-In-Chief, Journal of Mobile Technology in Medicine, ${ }^{2}$ Royal Eye \& Ear Hospital, Australia \\ Corresponding Author: rahul@journalmtm.com \\ Journal MTM 1:1:3-4, 2012 \\ doi:10.7309/jmtm.2
}

Modern medical practice is undergoing a transformation in the way it communicates and provides healthcare. The evolution in medicine has been advanced by the human desire to provide and receive a high standard of affordable medical care within an appropriate time. A significant contribution to this has been facilitated by advances in modern technology and telemedicine. Whilst a tsunami of easily accessible technology has engaged the attention of the medical community and improved access to medical care, particularly in remote areas, there is always an element of concern regarding safety, reliability, reproducibility and accuracy of telemedicine methods.

The strength of western medical practice is the establishment of evidence-based principles and guidelines. The concept of evidence-based medicine (EBM) is to merge clinical experience and patient values with the best available research. ${ }^{1}$ The purpose of this is not to create a rigid, automated framework that eliminates the clinician's judgement, but rather a logical framework that facilitates decision making that can be applied to the care of the individual patient. This concept of EBM is now entrenched in medical school curricula, such that junior doctors are taught to think in a more rigorous scientific framework while practicing the art of medicine.

Whilst the advent of telemedicine has the potential to improve access to patient assessment, treatment and monitoring, until such methods are validated and proven to be effective there is likely to be a degree reluctance on the part of medical fraternity to adopt these technologies in routine practice. In 2009, Thomas et al published a study of the use off-site display of patient parameters in several intensive care units (ICU), whereby clinicians located away from ICU were able to view patient parameters and make treatment decisions. ${ }^{2}$ At the conclusion of the study there was no significant difference in mortality and length of stay trends between the conventional and "trial" off-site telemedicine practice. Chen's article in the New York Times portrayed the polarized sentiment of doctors on this issue. ${ }^{3}$ Clearly some may feel the emergence of telemedicine alters the clinicianpatient relationship and raises concerns over patient safety. Conversely, another school of thought relates to practical considerations whereby remote access technology may facilitate timely care or referral for patients. In a vast continent such as Australia, the advent of radio communication was a critical milestone for the success of the Royal Flying Doctor Service (RFDS) in providing medical care to remote communities. At present, telemedicine accounts for one third of the 277,000 RDFS patient contacts per annum. ${ }^{4}$ These figures vindicate that modern medicine must adopt the principles of evidence based medicine in keeping up with the rising demand in order to assess what modalities of telemedicine are best suited to different environments.

The advances in technology and affordability of handheld devices have ensured that telemedicine and mobile technology will be an integral part of medical practice in the near future. In May 2011, the Manhattan Research Group survey of medical practitioners in the United States demonstrated that 75 per cent of physicians have purchased an Apple ${ }^{\circledR}$ Inc. mobile device such as iPad, iPhone, iPod. ${ }^{5}$ Prior research by the same group concluded that almost 30 per cent of doctors were using iPads to access patient records, radiology investigations, and communicating with their patients electronically. These trends reflect the rapidly growing demand for fast, readily accessible information in order to facilitate clinical decisions in a more efficient and timely manner. A search of the Apple "Appstore" reveals over 5300 medical, health and fitness applications. These range from medical reference resources for patients and clinicians, to medical calculators, examination instruments, and information storage programs across 
Fi. JMTM

a spectrum of medical disciplines. Yet as the market for such "apps" increases exponentially, one must be cautious regarding the quality and utility of these applications. This is particularly relevant to software that can be readily installed onto a mobile phone with the promise of converting it into an examination instrument, for example a chart to test colour vision. It can be argued that a natural process of censorship may occur as these applications can be rated for satisfaction by consumers. However, this merely equates to level five evidence. Thus, before such applications are adopted into routine practice clearly there needs to be rigorous scientific study of their validity.

The integration of accessible and affordable health technology is one of the six pillars of an effective health system as defined by the World Health Organisation. ${ }^{6}$ Mobile technology is arguably one of the most dynamic fields in medicine with the greatest potential to change clinical practice for the better. In order for it to be successfully integrated into practice, healthcare workers and patients need to be assured of its scientific validity. It is therefore imperative while we transition to the use of mobile technology, we do not compromise on the principles of evidence-based medicine when caring for our patients.

\section{References}

1. Craig JC, Irwig LM, Stockler MR. Evidence-based medicine: useful tools for decision making. Med J Aust 2001;174(5):248-53.
2. Thomas EJ, Lucke JF, Wueste L, Weavind L, Patel B. Association of telemedicine for remote monitoring of intensive care patients with mortality, complications, and length of stay. JAMA 2009;302(24):2671-8.

3. Chen PW. Are doctors ready for virtual visits? http://www.nytimes.com/2010/01/07/health/07chen.html. New York Times 2010 7/1/2010.

4. RFDS. Facts and Figures. http://www.flyingdoctor.org.au/About-Us/Facts--Figures/. In: Service RFD, editor: Royal Flying Doctor Service, 2011.

5. 75 percent of U.S. Physicians own some form of Apple device. http://manhattanresearch.com/News-and-

Events/Press-Releases/physician-iphone-ipad-adoption.

New York: Manhattan Research, 2011.

6. WHO. Strengthening health systems to improve health outcomes- WHO's Framework for Action. In: Organisation WH, editor. Geneva, 2007.

\section{Disclaimer}

All product names, logos, brands and other trademarks featured or referred to within the Journal of Mobile Technology in Medicine e-publication remain the property of their respective trademark holders.

These trademark holders are not affiliated with the Journal of Mobile Technology in Medicine, and do not sponsor or endorse this journal. 